\title{
Ignorance is Almost Bliss: Near-Optimal Stochastic Matching With Few Queries
}

\author{
AVRIM BLUM, Carnegie Mellon University \\ JOHN P. DICKERSON, Carnegie Mellon University \\ NIKA HAGHTALAB, Carnegie Mellon University \\ ARIEL D. PROCACCIA, Carnegie Mellon University \\ TUOMAS SANDHOLM, Carnegie Mellon University \\ ANKIT SHARMA ${ }^{1}$, Solvvy Inc.
}

\begin{abstract}
The stochastic matching problem deals with finding a maximum matching in a graph whose edges are unknown but can be accessed via queries. This is a special case of stochastic $k$-set packing, where the problem is to find a maximum packing of sets, each of which exists with some probability. In this paper, we provide edge and set query algorithms for these two problems, respectively, that provably achieve some fraction of the omniscient optimal solution.

Our main theoretical result for the stochastic matching (i.e., 2-set packing) problem is the design of an adaptive algorithm that queries only a constant number of edges per vertex and achieves a $(1-\epsilon)$ fraction of the omniscient optimal solution, for an arbitrarily small $\epsilon>0$. Moreover, this adaptive algorithm performs the queries in only a constant number of rounds. We complement this result with a non-adaptive (i.e., one round of queries) algorithm that achieves a $(0.5-\epsilon)$ fraction of the omniscient optimum. We also extend both our results to stochastic $k$-set packing by designing an adaptive algorithm that achieves a $\left(\frac{2}{k}-\epsilon\right)$ fraction of the omniscient optimal solution, again with only $O(1)$ queries per element. This guarantee is close to the best known polynomial-time approximation ratio of $\frac{3}{k+1}-\epsilon$ for the deterministic $k$-set packing problem [Fürer and $\mathrm{Yu} 2013$ ].

We empirically explore the application of (adaptations of) these algorithms to the kidney exchange problem, where patients with end-stage renal failure swap willing but incompatible donors. We show on both generated data and on real data from the first 169 match runs of the UNOS nationwide kidney exchange that even a very small number of non-adaptive edge queries per vertex results in large gains in expected successful matches.
\end{abstract}

Categories and Subject Descriptors: J.4 [Social and Behavioral Sciences]: Economics

General Terms: Algorithms, Economics, Experimentation

Additional Key Words and Phrases: Stochastic matching, stochastic $k$-set packing, kidney exchange

\section{INTRODUCTION}

In the stochastic matching problem, we are given an undirected graph $G=(V, E)$, where we do not know which edges in $E$ actually exist. Rather, for each edge $e \in E$, we are given an existence probability $p_{e}$. Of interest, then, are algorithms that first query some subset of edges to find the ones that exist, and based on these queries, produce a matching that is as large as possible. The stochastic matching problem is a special case of stochastic $k$-set packing, where each set exists only with some probability, and the problem is to find a packing of maximum size of those sets that do exist.

\footnotetext{
${ }^{1}$ This work was done while the author was a graduate student at Carnegie Mellon University.
}

This work was supported by the NSF under grants CCF-1101668, CCF-1116892, CCF-1215883, CCF1415460, IIS-0964579, IIS-1065251, IIS-1320620, and IIS-1350598, and by a National Defense Science \& Engineering Graduate Fellowship and a Sloan Research Fellowship. Authors' addresses: \{avrim, dickerson, nhaghtal, arielpro, sandholm, ankits\}@cs.cmu.edu .

Permission to make digital or hard copies of all or part of this work for personal or classroom use is granted without fee provided that copies are not made or distributed for profit or commercial advantage and that copies bear this notice and the full citation on the first page. Copyrights for components of this work owned by others than ACM must be honored. Abstracting with credit is permitted. To copy otherwise, or republish, to post on servers or to redistribute to lists, requires prior specific permission and/or a fee. Request permissions from permissions@acm.org.

EC'15, June 15-19, 2015, Portland, OR, USA. Copyright (c) 2015 ACM 978-1-4503-3410-5/15/06 ...\$15.00.

http://dx.doi.org/10.1145/2764468.2764479 
Without any constraints, one can simply query all edges or sets, and then output the maximum matching or packing over those that exist-but this level of freedom may not always be available. We are interested in the tradeoff between the number of queries and the fraction of the omniscient optimal solution achieved. Specifically, we ask: In order to perform as well as the omniscient optimum in the stochastic matching problem, do we need to query (almost) all the edges, that is, do we need a budget of $\Theta(n)$ queries per vertex, where $n$ is the number of vertices? Or, can we, for any arbitrarily small $\epsilon>0$, achieve a $(1-\epsilon)$ fraction of the omniscient optimum by using an $o(n)$ pervertex budget? We answer these questions, as well as their extensions to the $k$-set packing problem. We support our theoretical results empirically on both generated and real data from a large fielded kidney exchange in the United States.

\subsection{Our theoretical results and techniques}

Our main theoretical result gives a positive answer to the latter question for stochastic matching, by showing that, surprisingly, a constant per-vertex budget is sufficient to get $\epsilon$-close to the omniscient optimum. Indeed, we design a polynomial-time algorithm with the following properties: for any constant $\epsilon>0$, the algorithm queries at most $O(1)$ edges incident to any particular vertex, requires $O(1)$ rounds of parallel queries, and achieves a $(1-\epsilon)$ fraction of the omniscient optimum. ${ }^{2}$

The foregoing algorithm is adaptive, in the sense that its queries are conditioned on the answers to previous queries. Even though it requires only a constant number of rounds, it is natural to ask whether a non-adaptive algorithm-one that issues all its queries in one round-can also achieve a similar guarantee. We do not give a complete answer to this question, but we do present a nonadaptive algorithm that achieves a $0.5(1-\epsilon)$-approximation (for arbitrarily small $\epsilon>0$ ) to the omniscient optimum. We extend our matching results to a more general stochastic model in the full version of the paper. ${ }^{3}$

We extend our results to the stochastic $k$-set packing problem, where we are given a collection of sets, each with cardinality at most $k$. Stochastic Matching is a special case of Stochastic $k$-set packing: each set (which corresponds to an edge) has cardinality 2 , that is, $k=2$. In stochastic $k$-set packing, each set $s$ exists with some known probability $p_{s}$, and we need to query the sets to find whether they exist. Our objective is to output a collection of disjoint sets of maximum cardinality. We present adaptive and non-adaptive polynomial-time algorithms that achieve, for any constant $\epsilon>0$, at least $\left(\frac{2}{k}-\epsilon\right)$ and $(1-\epsilon) \frac{(2 / k)^{2}}{2 / k+1}$ fraction, respectively, of the omniscient optimum, again using $O(1)$ queries per element and hence $O(n)$ overall. For the sake of comparison, the best known polynomial-time algorithm for optimizing $k$-set packing in the standard non-stochastic setting has an approximation ratio of $\frac{3}{k+1}-\epsilon$ [Fürer and Yu 2013].

To better appreciate the challenge we face, we note that even in the stochastic matching setting, we do not have a clear idea of how large the omniscient optimum is. Indeed, there is a significant body of work on the expected cardinality of matching in complete random graphs (see, e.g., [Bollobás 2001, Chapter 7]), where the omniscient optimum is known to be close to $n$. But in our work we are dealing with arbitrary graphs where it can be a much smaller number. In addition, naïve algorithms fail to achieve our goal, even if they are allowed many queries. For example, querying a sublinear number of edges incident to each vertex, chosen uniformly at random, gives a vanishing fraction of the omniscient optimum - as we show in the full version of the paper.

The primary technical ingredient in the design of our adaptive algorithm is that if, in any round $r$ of the algorithm, the solution computed by round $r$ (based on previous queries) is small compared to the omniscient optimum, then the current structure must admit a large collection of disjoint constantsized 'augmenting' structures. These augmenting structures are composed of sets that have not been queried so far. Of course, we do not know whether these structures we are counting on to help

\footnotetext{
${ }^{2}$ This guarantee holds as long as all the non-zero $p_{e}$ 's are bounded away from zero by some constant. The constant can be arbitrarily small but should not depend on $n$.

${ }^{3}$ The full version of this paper is available at http://arxiv.org/abs/1407.4094
} 
augment our current matching actually exist; but we do know that these augmenting structures have constant size (and so each structure exists with some constant probability) and are disjoint (and therefore the outcomes of the queries to the different augmenting structures are independent). Hence, by querying all these structures in parallel in round $r$, in expectation, we can close a constant fraction of the gap between our current solution and the omniscient optimum. By repeating this argument over a constant number of rounds, we achieve a $(1-\epsilon)$ fraction of the omniscient optimum. In the case of stochastic matching, these augmenting structures are simply augmenting paths; in the more general case of $k$-set packing, we borrow the notion of augmenting structures from Hurkens and Schrijver [1989].

\subsection{Our experimental results: Application to kidney exchange}

Our work is directly motivated by applications to kidney exchange, a medical approach that enables kidney transplants. Transplanted kidneys are usually harvested from deceased donors; but as of April 26,2015 , there are 101,671 people on the US national waiting list, ${ }^{4}$ making the median waiting time dangerously long. Fortunately, kidneys are an unusual organ in that donation by living donors is also a possibility - as long as patients happen to be medically compatible with their potential donors.

In its simplest form-pairwise exchange-two incompatible donor-patient pairs exchange kidneys: the donor of the first pair donates to the patient of the second pair, and the donor of the second pair donates to the patient of the first pair. This setting can be represented as an undirected compatibility graph, where each vertex represents an incompatible donor-patient pair, and an edge between two vertices represents the possibility of a pairwise exchange. A matching in this graph specifies which exchanges take place.

The edges of the compatibility graph can be determined based on the medical characteristicsblood type and tissue type — of donors and patients. However, the compatibility graph only tells part of the story. Before a transplant takes place, a more accurate medical test known as a crossmatch test takes place. This test involves mixing samples of the blood of the patient and the donor (rather than simply looking up information in a database), making the test relatively costly and time consuming. Consequently, crossmatch tests are only performed for donors and patients that have been matched. While some patients are more likely to pass crossmatch tests than others-the probability is related to a measure of sensitization known as the person's Panel Reactive Antibody (PRA) - the average is as low as 30\% in major kidney exchange programs [Dickerson et al. 2013; Leishman et al. 2013]. This means that, if we tested a perfect matching over $n$ donor-patient pairs, we would expect only $0.09 n$ of the patients to actually receive a kidney. In contrast, the omniscient solution that runs crossmatch tests on all possible pairwise exchanges (in the compatibility graph) may be able to provide kidneys to all $n$ patients; but this solution is impractical.

Our adaptive algorithm for stochastic matching uncovers a sweet spot between these two extremes. On the one hand, it only mildly increases medical expenses, from one crossmatch test per patient, to a larger, yet constant, number; and it is highly parallelizable, requiring only a constant number of rounds, so the time required to complete all crossmatch tests does not scale with the number of donors and patients. On the other hand, the adaptive algorithm essentially recovers the entire benefit of testing all potentially feasible pairwise exchanges. The qualitative message of this theoretical result is clear: a mild increase in number of crossmatch tests provides nearly the full benefit of exhaustive testing.

The above discussion pertains to pairwise kidney exchange. However, modern kidney exchange programs regularly employ swaps involving three donor-patient pairs, which are known to provide significant benefit compared to pairwise swaps alone [Roth et al. 2007; Ashlagi and Roth 2014]. Mathematically, we can consider a directed graph, where an edge $(u, v)$ means that the donor of pair $u$ is compatible with the patient of pair $v$ (before a crossmatch test was performed). In this graph, pairwise and 3-way exchanges correspond to 2-cycles and 3-cycles, respectively. Our adaptive algorithm for 3 -set packing then provides a (2/3)-approximation to the omniscient optimum,

\footnotetext{
${ }^{4}$ http://optn.transplant.hrsa.gov
} 
using only $O(1)$ crossmatch tests per patient and $O(n)$ overall. While the practical implications of this result are currently not as crisp as those of its pairwise counterpart, future work may improve the approximation ratio (using $O(n)$ queries and an exponential-time algorithm), as we explain in Section 8.1.

To bridge the gap between theory and practice, we provide experiments on both simulated data and real data from the first 169 match runs of the United Network for Organ Sharing (UNOS) US nationwide kidney exchange, which now includes 143 transplant centers-approximately $60 \%$ of the transplant centers in the US. The exchange began matching in October 2010 and now matches on a biweekly basis. Using adaptations of the algorithms presented in this paper, we show that even a small number of non-adaptive rounds, followed by a single period during which only those edges selected during those rounds are queried, results in large gains relative to the omniscient matching. We discuss the policy implications of this promising result in Section 8.2.

\section{RELATED WORK}

While papers on stochastic matching often draw on kidney exchange for motivation-or at least mention it in passing - these two research areas are almost disjoint. We therefore discuss them separately in Sections 2.1 and 2.2.

\subsection{Stochastic matching}

Prior work has considered multiple variants of stochastic matching. A popular variant is the querycommit problem, where the algorithm is forced to add any queried edge to the matching if the edge is found to exist. Goel and Tripathi [2012] establish an upper bound of 0.7916 for graphs in which no information is available about the edges, while Costello et al. [2012] establish a lower bound of 0.573 and an upper bound of 0.898 for graphs in which each edge $e$ exists with a given probability $p_{e}$. Similarly to our work, these approximation ratios are with respect to the omniscient optimum, but the informational disadvantage of the algorithm stems purely from the query-commit restriction.

Within the query-commit setting, another thread of work [Chen et al. 2009; Adamczyk 2011; Bansal et al. 2012] imposes an additional per-vertex budget constraint where the algorithm is not allowed to query more than a specified number, $b_{v}$, of edges incident to vertex $v$. With this additional constraint, the benchmark that the algorithm is compared to switches from the omniscient optimum to the constrained optimum, i.e., the performance of the best decision tree that obeys the per-vertex budget constraints and the query-commit restriction. In other words, the algorithm's disadvantage compared to the benchmark is only that it is constrained to run in polynomial time. Here, again, the best known approximation ratios are constant. A generalization of these results to packing problems has been studied by Gupta and Nagarajan [2013].

Similarly to our work, Blum et al. [2013] consider a stochastic matching setting without the query-commit constraint. They set the per-vertex budget to exactly 2 , and ask which subset of edges is queried by the optimal collection of queries subject to this constraint. They prove structural results about the optimal solution, which allow them to show that finding the optimal subset of edges to query is NP-hard. In addition, they give a polynomial-time algorithm that finds an almost optimal solution on a class of random graphs (inspired by kidney exchange settings). Crucially, the benchmark of Blum et al. [2013] is also constrained to two queries per vertex.

There is a significant body of work in stochastic optimization more broadly, for instance, the papers of Dean et al. [2004] (Stochastic Knapsack), Gupta et al. [2012] (Stochastic Orienteering), and Asadpour et al. [2008] (Stochastic submodular maximization).

\subsection{Kidney exchange}

Early models of kidney exchange did not explicitly consider the setting where an edge that is chosen to be matched only exists probabilistically. Recent research by Dickerson et al. [2013] and Anderson et al. [2015b] focuses on the kidney exchange application and restricts attention to a single crossmatch test per patient (the current practice), with a similar goal of maximizing the expected number of matched vertices, in a realistic setting (for example, they allow 3-cycles and chains ini- 
tiated by altruistic donors, who enter the exchange without a paired patient). They develop integer programming techniques, which are empirically evaluated using real and synthetic data. Manlove and O'Malley [2015] discuss the integer programming formulation used by the national exchange in the United Kingdom, which takes edge failures into account in an ad hoc way by, for example, preferring shorter cycles to longer ones. To our knowledge, our paper is the first to describe a general method for testing any number of edges before the final match run is performed-and to provide experiments on real data showing the expected effect on fielded exchanges of such edge querying policies.

Another form of stochasticity present in fielded kidney exchanges is the arrival and departure of donor-patient pairs over time (and the associated arrival and departure of their involved edges in the compatibility graph). Recent work has addressed this added form of dynamism from a theoretical [Ünver 2010; Akbarpour et al. 2014; Anderson et al. 2015a] and experimental [Awasthi and Sandholm 2009; Dickerson et al. 2012a; Dickerson and Sandholm 2015] point of view. Theoretical models have not addressed the case where an edge in the current graph may not exist (as we do in this paper); the more recent experimental papers have incorporated this possibility, but have not considered the problem of querying edges before recommending a final matching. We leave as future research the analysis of edge querying in stochastic matching in such a dynamic model.

\section{THE MODEL}

For any graph $G=(V, E)$, let $M(E)$ denote its maximum (cardinality) matching. ${ }^{5}$ In addition, for two matchings $M$ and $M^{\prime}$, we denote their symmetric difference by $M \Delta M^{\prime}=\left(M \cup M^{\prime}\right) \backslash(M \cap$ $\left.M^{\prime}\right)$; it includes only paths and cycles consisting of alternating edges of $M$ and $M^{\prime}$.

In the stochastic setting, given a set of edges $X$, define $X_{p}$ to be the random subset formed by including each edge of $X$ independently with probability $p$. We will assume for ease of exposition that $p_{e}=p$ for all edges $e \in E$. Our results hold when $p$ is a lower bound, i.e., $p_{e} \geq p$ for all $e \in E$. Furthermore, in the full version of this paper, we show that we can extend our results to a more general setting where the existence probabilities of edges incident to any particular vertex are correlated.

Given a graph $G=(V, E)$, define $\bar{M}(E)$ to be $\mathbb{E}\left[\left|M\left(E_{p}\right)\right|\right]$, where the expectation is taken over the random draw $E_{p}$. In addition, given the results of queries on some set of edges $T$, define $\bar{M}(E \mid T)$ to be $\mathbb{E}\left[\left|M\left(X_{p} \cup T^{\prime}\right)\right|\right]$, where $T^{\prime} \subseteq T$ is the subset of edges of $T$ that are known to exist based on the queries, and $X=E \backslash T$.

In the non-adaptive version of our problem, the goal is to design an algorithm that, given a graph $G=(V, E)$ with $|V|=n$, queries a subset $X$ of edges in parallel such that $|X|=O(n)$, and maximizes the ratio $\bar{M}(X) / \bar{M}(E)$.

In contrast, an adaptive algorithm proceeds in rounds, and in each round queries a subset of edges in parallel. Based on the results of the queries up to the current round, it can choose the subset of edges to test in the next round. Formally, an R-round adaptive stochastic matching algorithm selects, in each round $r$, a subset of edges $X_{r} \subseteq E$, where $X_{r}$ can be a function of the results of the queries on $\bigcup_{i<r} X_{i}$. The objective is to maximize the ratio $\mathbb{E}\left[\left|M\left(\bigcup_{1 \leq i \leq R} X_{i}\right)\right|\right] / \bar{M}(E)$, where the expectation in the numerator is taken over the outcome of the query results and the sets $X_{i}$ chosen by the algorithm.

To gain some intuition for our goal of arbitrarily good approximations to the omniscient optimum, and why it is challenging, let us consider a naïve algorithm and understand why it fails. This nonadaptive algorithm schedules $R=O(\log (n) / p)$ queries for each vertex as follows. First, order all vertices arbitrarily and start with an empty set of queries. In order, for each vertex $v$, let $N_{R}(v)$ be the set of neighbors of $v$ for whom at most $R$ queries have been scheduled. Schedule $\min \left\{R, N_{R}(v)\right\}$

\footnotetext{
${ }^{5}$ In the notation $M(E)$, we intentionally suppress the dependence on the vertex set $V$, since we care about the maximum matchings of different subsets of edges for a fixed vertex set.
} 
queries, each between $v$ and an element of $N_{R}(v)$, where these elements are selected uniformly at random from $N_{R}(v)$.

The next example shows that this proposed algorithm only achieves $\frac{5}{6}$ fraction of the omniscient optimal solution, as opposed to our goal of achieving arbitrarily good $(1-\epsilon)$ approximations to the omniscient optimal. Furthermore, in the following example when each edge exists with probability $p>\frac{5}{6}$, this algorithm still only achieves a $\frac{5}{6}$ fraction of the omniscient optimal solution, which is worse than a trivial algorithm of just picking one maximum matching that guarantees a matching of size $p n$.

Example 3.1. Consider the graph $G=(V, E)$ whose vertices are partitioned into sets $A, B, C$, and $D$, such that $|A|=|B|=\frac{n}{2}$ and $|C|=|D|=n$. Let $E$ consist of two random bipartite graphs of degree $R=O(\log (n) / p)$ between $A$ and $B$ and similarly between $C$ and $D$. And let $B$ and $C$ be connected with a complete bipartite graph. Let $p$ be the existence probability of any edge.

With high probability, there is a perfect matching that matches $A$ to $B$ and $C$ to $D$. However, by the time the algorithm has processed half of the vertices, expected half of the vertices in $A, B, C$, and $D$ are processed. For every vertex in $B$, this vertex has more neighbors in $C$ than in $D$. So, at this point, with high probability all vertices of $B$ already have $R$ queries scheduled from half of the vertices in $C$. Therefore, after this point in the algorithm, no edges between $A$ and $B$ will be queried. So, $\frac{1}{2}$ of the vertices in $A$ remain unmatched. Compared to the omniscient optimum-which is a perfect matching with high probability - the approximation ratio of this algorithm is at most $\frac{5}{6}$.

For an analysis of additional naïve algorithms, please refer to the full version of the paper.

\section{ADAPTIVE ALGORITHM: $(1-\epsilon)$-APPROXIMATION}

In this section, we present our main result: an adaptive algorithm — formally given as Algorithm 1that achieves a $(1-\epsilon)$ approximation to the omniscient optimum for arbitrarily small $\epsilon>0$, using $O(1)$ queries per vertex and $O(1)$ rounds.

The algorithm is initialized with the empty matching $M_{0}$. At the end of each round $r$, our goal is to maintain a maximum matching $M_{r}$ on the set of edges that are known to exist (based on queries made so far). To this end, at round $r$, we compute the maximum matching $O_{r}$ on the set of edges that are known to exist and the ones that have not been queried yet (Step 2a). We consider augmenting paths in $O_{r} \Delta M_{r-1}$, and query all the edges in them (Steps $2 \mathrm{~b}$ and 2c). Based on the results of these queries $\left(Q_{r}\right)$, we update the maximum matching $\left(M_{r}\right)$. Finally, we return the maximum matching $M_{R}$ computed after $R=\frac{\log (2 / \epsilon)}{p^{2 / \epsilon}}$ rounds. (Let us assume that $R$ is an integer for ease of exposition.)

Algorithm 1 Adaptive Algorithm for Stochastic Matching: $(1-\epsilon)$ Approximation Input: A graph $G=(V, E)$.

Parameter: $R=\frac{\log (2 / \epsilon)}{p^{2 / \epsilon}}$

(1) Initialize $M_{0}$ to the empty matching and $W_{1} \leftarrow \emptyset$.

(2) For $r=1, \ldots, R$, do

(a) Compute a maximum matching, $O_{r}$, in $\left(V, E \backslash W_{r}\right)$.

(b) Set $Q_{r}$ to the collection of all augmenting paths of $M_{r-1}$ in $O_{r} \Delta M_{r-1}$.

(c) Query the edges in $Q_{r}$. Let $Q_{r}^{\prime}$ and $Q_{r}^{\prime \prime}$ represent the set of existing and non-existing edges.

(d) $W_{r+1} \leftarrow W_{r} \cup Q_{r}^{\prime \prime}$.

(e) Set $M_{r}$ to the maximum matching in $\left(V, \bigcup_{j=1}^{r} Q_{j}^{\prime}\right)$.

(3) Output $M_{R}$.

It is easy to see that this algorithm queries at most $\frac{\log (2 / \epsilon)}{p^{2 / \epsilon}}$ edges per vertex: In a given round $r$, the algorithm queries edges that are in augmenting paths of $O_{r} \Delta M_{r-1}$. Since there is at most one augmenting path using any particular vertex, the algorithm queries at most one edge per vertex in 
each round. Furthermore, the algorithm executes $\frac{\log (2 / \epsilon)}{p^{2 / \epsilon}}$ rounds. Therefore, the number of queries issued by the algorithm per vertex is as claimed.

The rest of the section is devoted to proving that the matching returned by this algorithm after $R$ rounds has cardinality that is, in expectation, at least a $(1-\epsilon)$ fraction of $\bar{M}(E)$.

THEOREM 4.1. For any graph $G=(V, E)$ and any $\epsilon>0$, Algorithm 1 returns a matching whose expected cardinality is at least $(1-\epsilon) \bar{M}(E)$ in $R=\frac{\log (2 / \epsilon)}{p^{(2 / \epsilon)}}$ rounds.

As mentioned in Section 1, one of the insights behind this result is the existence of many disjoint augmenting paths of bounded length that can be used to augment a matching that is far from the omniscient optimum, that is, a lower bound on the number of elements in $Q_{r}$ of a given length $L$. This observation is formalized in the following lemma. (We emphasize that the lemma pertains to the non-stochastic setting.)

LEMMA 4.2. Consider a graph $G=(V, E)$ with two matchings $M_{1}$ and $M_{2}$. Suppose $\left|M_{2}\right|>$ $\left|M_{1}\right|$. Then in $M_{1} \Delta M_{2}$, for any odd length $L \geq 1$, there exist at least $\left|M_{2}\right|-\left(1+\frac{2}{L+1}\right)\left|M_{1}\right|$ augmenting paths of length at most $L$, which augment the cardinality of $M_{1}$.

PROOF. Let $x_{l}$ be the number of augmenting paths of length $l$ (for any odd $l \geq 1$ ) found in $M_{1} \Delta M_{2}$ that augment the cardinality of $M_{1}$. Each augmenting path increases the size of $M_{1}$ by 1 , so the total number of augmenting paths $\sum_{l \geq 1} x_{l}$ is at least $\left|M_{2}\right|-\left|M_{1}\right|$. Moreover, each augmenting path of length $l$ has $\frac{l-1}{2}$ edges in $M_{1}$. Hence, $\sum_{l \geq 1} \frac{l-1}{2} x_{l} \leq\left|M_{1}\right|$. In particular, this implies that $\frac{L+1}{2} \sum_{l \geq L+2} x_{l} \leq\left|M_{1}\right|$. We conclude that

$$
\sum_{l=1}^{L} x_{l}=\sum_{l \geq 1} x_{l}-\sum_{l \geq L+2} x_{l} \geq\left(\left|M_{2}\right|-\left|M_{1}\right|\right)-\frac{2}{L+1}\left|M_{1}\right|=\left|M_{2}\right|-\left(1+\frac{2}{L+1}\right)\left|M_{1}\right| .
$$

The rest of the theorem's proof requires some additional notation. At the beginning of any given round $r$, the algorithm already knows about the existence (or non-existence) of the edges in $\bigcup_{i=1}^{r-1} Q_{i}$. We use $Z_{r}$ to denote the expected size of the maximum matching in graph $G=(V, E)$ given the results of the queries $\bigcup_{i=1}^{r-1} Q_{i}$. More formally, $Z_{r}=\bar{M}\left(E \mid \bigcup_{i=1}^{r-1} Q_{i}\right)$. Note that $Z_{1}=\bar{M}(E)$.

For a given $r$, we use the notation $\mathbb{E}_{Q_{r}}[X]$ to denote the expected value of $X$ where the expectation is taken only over the outcome of query $Q_{r}$, and fixing the outcomes on the results of queries $\bigcup_{i=1}^{r-1} Q_{i}$. Moreover, for a given $r$, we use $\mathbb{E}_{Q_{r}, \ldots, Q_{R}}[X]$ to denote the expected value of $X$ with the expectation taken over the outcomes of queries $\bigcup_{i=r}^{R} Q_{i}$, and fixing an outcome on the results of queries $\bigcup_{i=1}^{r-1} Q_{i}$.

In Lemma 4.3, for any round $r$ and for any outcome of the queries $\bigcup_{i=1}^{r-1} Q_{i}$, we lower-bound the expected increase in the size of $M_{r}$ over the size of $M_{r-1}$, with the expectation being taken only over the outcome of edges in $Q_{r}$. This lower bound is a function of $Z_{r}$.

Lemma 4.3. For any $r \in[R]$, odd $L$, and $Q_{1}, \cdots, Q_{r-1}$, it holds that $\mathbb{E}_{Q_{r}}\left[\left|M_{r}\right|\right] \geq(1-$ $\gamma)\left|M_{r-1}\right|+\alpha Z_{r}$, where $\gamma=p^{(L+1) / 2}\left(1+\frac{2}{L+1}\right)$ and $\alpha=p^{(L+1) / 2}$.

Proof. By Lemma 4.2, there exist at least $\left|O_{r}\right|-\left(1+\frac{2}{L+1}\right)\left|M_{r-1}\right|$ augmenting paths in $O_{r} \Delta M_{r-1}$ that augment $M_{r-1}$ and are of length at most $L$. The $O_{r}$ part of every augmenting path of length at most $L$ exists independently with probability at least $p^{(L+1) / 2}$. Therefore, the expected 
increase in the size of the matching is:

$$
\begin{aligned}
\mathbb{E}_{Q_{r}}\left[\left|M_{r}\right|\right]-\left|M_{r-1}\right| & \geq p^{\frac{L+1}{2}}\left(\left|O_{r}\right|-\left(1+\frac{2}{L+1}\right)\left|M_{r-1}\right|\right) \\
& =\alpha\left|O_{r}\right|-\gamma\left|M_{r-1}\right| \geq \alpha Z_{r}-\gamma\left|M_{r-1}\right|,
\end{aligned}
$$

where the last inequality holds by the fact that $Z_{r}$, which is the expected size of the optimal matching with expectation taken over non-queried edges, cannot be larger than $O_{r}$, which is the maximum matching assuming that every non-queried edge exists.

We are now ready to prove the theorem.

Proof OF THEOREM 4.1. Let $L=\frac{4}{\epsilon}-1$; it is assumed to be an odd integer for ease of exposition. ${ }^{6}$ By Lemma 4.3, we know that for every $r \in[R], \mathbb{E}_{Q_{r}}\left[\left|M_{r} \| \geq(1-\gamma)\right| M_{r-1} \mid+\alpha Z_{r}\right.$, where $\gamma=p^{(L+1) / 2}\left(1+\frac{2}{L+1}\right)$, and $\alpha=p^{(L+1) / 2}$. We will use this inequality repeatedly to derive our result. We will also require the equality

$$
\mathbb{E}_{Q_{r-1}}\left[Z_{r}\right]=\mathbb{E}_{Q_{r-1}}\left[\bar{M}\left(E \mid \bigcup_{i=1}^{r-1} Q_{i}\right)\right]=\bar{M}\left(E \mid \bigcup_{i=1}^{r-2} Q_{i}\right)=Z_{r-1} .
$$

First, applying Lemma 4.3 at round $R$, we have that $\mathbb{E}_{Q_{R}}\left[\left|M_{R}\right|\right] \geq(1-\gamma)\left|M_{R-1}\right|+\alpha Z_{R}$. This inequality is true for any fixed outcomes of $Q_{1}, \ldots, Q_{R-1}$. In particular, we can take the expectation over $Q_{R-1}$, and obtain

$$
\mathbb{E}_{Q_{R-1}, Q_{R}}\left[\left|M_{R}\right|\right] \geq(1-\gamma) \mathbb{E}_{Q_{R-1}}\left[\left|M_{R-1}\right|\right]+\alpha \mathbb{E}_{Q_{R-1}}\left[Z_{R}\right] .
$$

By Equation (1), we know that $\mathbb{E}_{Q_{R-1}}\left[Z_{R}\right]=Z_{R-1}$. Furthermore, we can apply Lemma 4.3 to $\mathbb{E}_{Q_{R-1}}\left[\left|M_{R-1}\right|\right]$ to get the following inequality:

$$
\begin{aligned}
\mathbb{E}_{Q_{R-1}, Q_{R}}\left[\left|M_{R}\right|\right] & \geq(1-\gamma) \mathbb{E}_{Q_{R-1}}\left[\left|M_{R-1}\right|\right]+\alpha \mathbb{E}_{Q_{R-1}}\left[Z_{R}\right] \\
& \geq(1-\gamma)\left((1-\gamma)\left|M_{R-2}\right|+\alpha Z_{R-1}\right)+\alpha Z_{R-1} \\
& =(1-\gamma)^{2}\left|M_{R-2}\right|+\alpha(1+(1-\gamma)) Z_{R-1} .
\end{aligned}
$$

We repeat the above steps by sequentially taking expectations over $Q_{R-2}$ through $Q_{1}$, and at each step applying Equation (1) and Lemma 4.3. This gives us

$$
\begin{aligned}
\mathbb{E}_{Q_{1}, \ldots, Q_{R}}\left[\left|M_{R}\right|\right] & \geq(1-\gamma)^{R}\left|M_{0}\right|+\alpha\left(1+(1-\gamma)+\cdots+(1-\gamma)^{R-1}\right) Z_{1} \\
& =\alpha \frac{1-(1-\gamma)^{R}}{\gamma} Z_{1},
\end{aligned}
$$

where the second transition follows from the initialization of $M_{0}$ as an empty matching. Since $L=\frac{4}{\epsilon}-1$ and $R=\frac{\log (2 / \epsilon)}{p^{2 / \epsilon}}$, we have

$$
\frac{\alpha}{\gamma}\left(1-(1-\gamma)^{R}\right)=\left(1-\frac{2}{L+1}\right)\left(1-(1-\gamma)^{R}\right) \geq 1-\frac{2}{L+1}-e^{-\gamma R} \geq 1-\frac{\epsilon}{2}-\frac{\epsilon}{2}=1-\epsilon,
$$

where the second transition is true because $e^{-x} \geq 1-x$ for all $x \in \mathbb{R}$. We conclude that $\mathbb{E}_{Q_{1}, \ldots, Q_{R}}\left[\left|M_{R}\right|\right] \geq(1-\epsilon) Z_{1}$. Because $Z_{1}=\bar{M}(E)$, it follows that the expected size of the algorithm's output is at least $(1-\epsilon) \bar{M}(E)$.

\footnotetext{
${ }^{6}$ Otherwise there exists $\epsilon / 2 \leq \epsilon^{\prime} \leq \epsilon$ such that $\frac{4}{\epsilon^{\prime}}-1$ is an odd integer. We use a similar simplification in the proofs of other results in the full version of this paper.
} 
In the full version of this paper, we extend our results to the setting where edges have correlated existence probabilities - an edge's probability is determined by parameters associated with its two vertices. This generalization gives a better model for kidney exchange, as some patients are highly sensitized and therefore harder to match in general; this means that all edges incident to such vertices are less likely to exist. We consider two settings, first, where an adversary chooses the vertex parameters, and second, where these parameters are drawn from a distribution. Our approach involves excluding from our analysis edges whose existence probability is too low. We do so by showing that (under specific conditions) excluding any augmenting path that includes such edges still leaves us with a large number of constant-size augmenting paths.

\section{NON-ADAPTIVE ALGORITHM: 0.5-APPROXIMATION}

The adaptive algorithm, Algorithm 1, augments the current matching by computing a maximum matching on queried edges that are known to exist, and edges that have not been queried. One way to extend this idea to the non-adaptive setting is the following: we can simply choose several edge-disjoint matchings, and hope that they help in augmenting each other. In this section, we ask: How close can this non-adaptive interpretation of our adaptive approach take us to the omniscient optimum?

In more detail, our non-adaptive algorithm-formally given as Algorithm 2-iterates $R=$ $\frac{\log (2 / \epsilon)}{p^{2 / \epsilon}}$ times. In each iteration, it picks a maximum matching and removes it. The set of edges queried by the algorithm is the union of the edges chosen in some iteration. We will show that, for any arbitrarily small $\epsilon>0$, the algorithm finds a $0.5(1-\epsilon)$-approximate solution. Since we allow an arbitrarily small (though constant) probability $p$ for stochastic matching, achieving a 0.5 approximation independently of the value of $p$, while querying only a linear number of edges, is nontrivial. For example, a naïve algorithm that only queries one maximum matching clearly does not guarantee a 0.5 -approximation-it would guarantee only a $p$-approximation. In addition, the example given in Section 3 shows that choosing edges at random performs poorly.

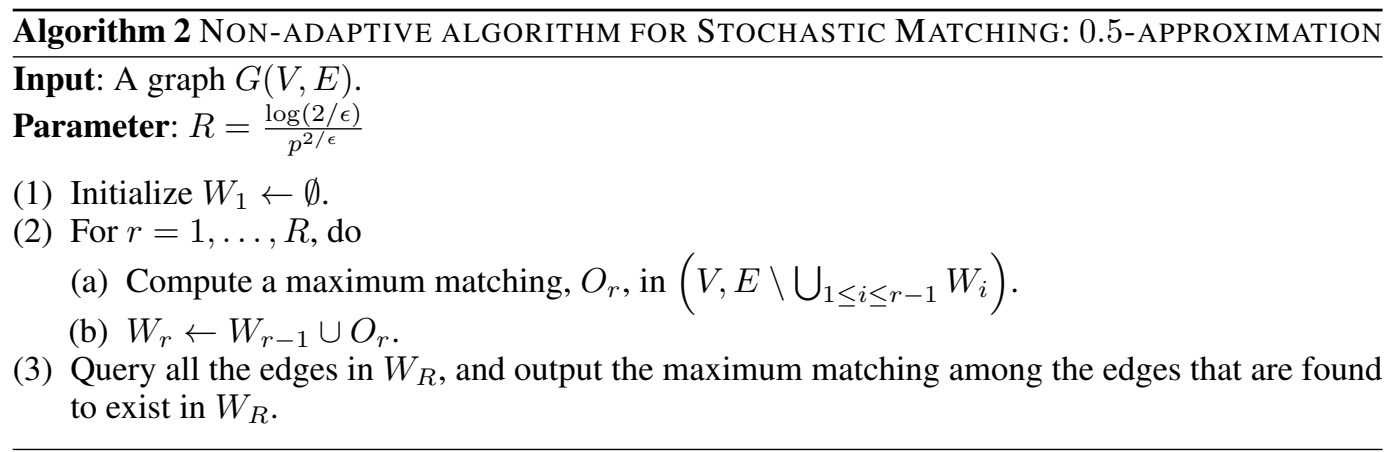

The number of edges incident to any particular vertex that are queried by the algorithm is at most $\frac{\log (2 / \epsilon)}{p^{2 / \epsilon}}$, because the vertex can be matched with at most one neighbor in each round. The next theorem, whose proof appears in the full version of the paper, establishes the approximation guarantee of Algorithm 2.

THEOREM 5.1. Given a graph $G=(V, E)$ and any $\epsilon>0$, the expected size $\bar{M}\left(W_{R}\right)$ of the matching produced by Algorithm 2 is at least a $0.5(1-\epsilon)$ fraction of $\bar{M}(E)$.

As explained in Section 8.1, we do not know whether in general non-adaptive algorithms can achieve a $(1-\epsilon)$-approximation with $O(1)$ queries per vertex. However, if there is such an algorithm, it is not Algorithm 2! Indeed, the next theorem (whose proof appears in the full version of the paper) shows that the algorithm cannot give an approximation ratio better than $5 / 6$ to the omniscient optimum. This fact holds even when $R=\Theta(\log n)$. 
THEOREM 5.2. Let $p=0.5$. For any $\epsilon>0$ there exists $n$ and $a$ graph $(V, E)$ with $|V| \geq n$ such that Algorithm 2, with $R=O(\log n)$, returns a matching with expected size of at most $\frac{5}{6} \bar{M}(E)+\epsilon$.

Despite this somewhat negative result, in Section 7, we show experimentally on realistic kidney exchange compatibility graphs that Algorithm 2 performs very well for even very small values of $R$, across a wide range of values of $p$.

\section{GENERALIZATION TO $K$-SET PACKING}

So far we have focused on stochastic matching, for ease of exposition. But our approach directly generalizes to the $k$-set packing problem. Here we describe this extension for the adaptive (Section 6.1) and non-adaptive (Section 6.2) cases, and relegate the details - in particular, most proofsto the full version of the paper.

Formally, a $k$-set packing instance $(U, A)$ consists of a set of elements $U,|U|=n$, and a collection of subsets $A$, such that each subset $S$ in $A$ contains at most $k$ elements of $U$, that is, $S \subseteq U$ and $|S| \leq k$. Given such an instance, a feasible solution is a collection of sets $B \subseteq A$ such that any two sets in $B$ are disjoint. We use $K(A)$ to denote the largest feasible solution $B$.

Finding an optimal solution to the $k$-set packing problem is NP-hard (see, e.g., [Abraham et al. 2007] for the special case of $k$-cycle packing). Hurkens and Schrijver [1989] designed a polynomialtime local search algorithm with an approximation ratio of $\left(\frac{2}{k}-\eta\right)$, using local improvements of constant size that depends only on $\eta$ and $k$. We denote this constant by $s_{\eta, k}$. More formally, consider an instance $(U, A)$ of $k$-set packing and let $B \subseteq A$ be a collection of disjoint $k$-sets. $(C, D)$ is said to be an augmenting structure for $B$ if removing $D$ and adding $C$ to $B$ increases the cardinality and maintains the disjointness of the resulting collection, i.e., if $(B \cup C) \backslash D$ is a disjoint collection of $k$-sets and $|(B \cup C) \backslash D|>|B|$, where $C \subseteq A$ and $D \subseteq B$.

Hurkens and Schrijver [1989] have also shown that an approximation ratio better than $2 / k$ cannot be achieved with structures of constant size. While subsequent work [Fürer and Yu 2013] has improved the approximation ratio, their local search algorithm finds structures of super-constant size. This is inconsistent with our technical approach, as we need each queried structure to exist (in its entirety) with constant probability.

To be more precise, Hurkens and Schrijver [1989] prove:

LEMMA 6.1 ([HURKENS AND SCHRIJVER 1989]). Given a collection $B$ of disjoint sets such that $|B|<(2 / k-\eta)|K(A)|$, there exists an augmenting structure $(C, D)$ for $B$ such that both $C$ and $D$ have at most $s_{\eta, k}$ sets, for a constant $s_{\eta, k}$ that depends only on $\eta$ and $k$.

However, we need to find many augmenting structures. We use Lemma 6.1 to prove:

LEMMA 6.2. If $|B|<|K(A)|$, then there exist $\frac{1}{k s_{\eta, k}}\left(|K(A)|-\frac{|B|}{\frac{2}{k}-\eta}\right)$ disjoint augmenting structures that augment the cardinality of $B$, each with size at most $s_{\eta, k}$. Moreover, this collection of augmenting structures can be found in polynomial time.

PROOF. We prove the lemma using Algorithm 3. We claim that if we run this algorithm on the $k$-set packing instance $(U, A)$ and the collection $B$, then it will return a collection $Q$ of at least $T=\frac{1}{k s_{\eta, k}}\left(|K(A)|-\frac{|B|}{\frac{2}{k}-\eta}\right)$ disjoint augmenting structures $(C, D)$ for $B$. By Step 2c, we are guaranteed that $Q$ consists of disjoint augmenting structures. Hence, all that is left to show is that in each of the first $T$ iterations, at Step 2a, we are able to find a nonempty augmenting structure $(C, D)$ for $B$.

By Lemma 6.1, we know that if at iteration $t$ it is the case that $|B|<\left(\frac{2}{k}-\eta\right)\left|K\left(A_{t}\right)\right|$, then we will find an augmenting structure $(C, D)$ of size $s_{\eta, k}$ for $B$. To prove that the inequality holds at each iteration $t \leq T$, we first claim that for all $t$,

$$
\left|K\left(A_{t}\right)\right| \geq|K(A)|-(t-1) \cdot k \cdot s_{\eta, k}
$$


We prove this by induction. The claim is clearly true for the base case of $t=1$. For the inductive step, suppose it is true for $t$, then we know that $\left|K\left(A_{t}\right)\right| \geq|K(A)|-(t-1) \cdot k \cdot s_{\eta, k}$. At iteration $t$, the augmenting structure $(C, D)$ can intersect with at most $s_{\eta, k} \cdot k$ sets of $K\left(A_{t}\right)$. This is true since $K\left(A_{t}\right)$ consists of disjoint sets, and the augmenting structure $(C, D)$ is of size at most $s_{\eta, k}$. Hence, Step 2c reduces $\left|K\left(A_{t}\right)\right|$ by at most $k \cdot s_{\eta, k}$. So, $\left|K\left(A_{t+1}\right)\right| \geq\left|K\left(A_{t}\right)\right|-k \cdot s_{\eta, k}$. Combining the two inequalities, $\left|K\left(A_{t}\right)\right| \geq|K(A)|-(t-1) \cdot k \cdot s_{\eta, k}$ and $\left|K\left(A_{t+1}\right)\right| \geq\left|K\left(A_{t}\right)\right|-k \cdot s_{\eta, k}$, we have $\left|K\left(A_{t+1}\right)\right| \geq|K(A)|-t \cdot k \cdot s_{\eta, k}$. This establishes Equation (3).

We conclude that if the for-loop adds non-empty augmenting structures only for the first $t$ rounds, it must be the case that $|B| \geq\left(\frac{2}{k}-\eta\right)\left|K\left(A_{t+1}\right)\right|$, and therefore $|B| \geq\left(\frac{2}{k}-\eta\right)\left(|K(A)|-t \cdot k \cdot s_{\eta, k}\right)$ which implies that $t \geq \frac{1}{k s_{\eta, k}}\left(|K(A)|-\frac{|B|}{\frac{2}{k}-\eta}\right)$.

Algorithm 3 Finding CONSTANT-SIZE DISJOINT AUGMENTING STRUCTURES FOR $k$-SETS

Input: $k$-set packing instance $(U, A)$ and a collection $B \subseteq A$ of disjoint sets.

Output: Collection $Q$ of disjoint augmenting structures $(C, D)$ for $B$.

Parameter: $s_{\eta, k}$ (the desired maximum size of the augmenting structures)

(1) Initialize $A_{1} \leftarrow A$ and $Q \leftarrow \emptyset$ (empty set).

(2) For $t=1, \cdots,|A|$

(a) Find an augmenting structure $(C, D)$ of size $s_{\eta, k}$ for $B$ on the $k$-set instance $\left(U, A_{t}\right)$.

(b) Add $(C, D)$ to $Q$. (If $C$ is an empty set, break out of the loop.)

(c) Set $A_{t+1}$ to be $A_{t}$ minus the collection $C$ and any set in $A_{t} \backslash B$ that intersects with $C$.

(3) Output $Q$.

\subsection{Adaptive algorithm for $k$-set packing}

Turning to the stochastic version of the problem, given $(U, A)$, let $A_{p}$ be a random subset of $A$ where each set from $A$ is included in $A_{p}$ independently with probability $p$. We then define $\bar{K}(A)$ to be $\mathbb{E}\left[\left|K\left(A_{p}\right)\right|\right]$, where the expectation is taken over the random draw $A_{p}$. Similarly to the matching setting, this is the omniscient optimum-our benchmark.

We extend the ideas introduced earlier in the paper for matching, together with Lemma 6.2 and additional ingredients, to obtain the following result for the adaptive problem.

THEOREM 6.3. There exists an adaptive polynomial-time algorithm that, given a $k$-set instance $(U, A)$ and $\epsilon>0$, uses $O(1)$ rounds and $O(n)$ queries overall, and returns a set $B_{R}$ whose expected cardinality is at least a $(1-\epsilon) \frac{2}{k}$ fraction of $\bar{K}(A)$.

With an eye toward Theorem 6.3, Algorithm 4 is a polynomial-time algorithm that can be used to find such a packing that approximates the omniscient optimum. In each round $r$, the algorithm maintains a feasible $k$-set packing $B_{r}$ based on the $k$-sets that have been queried so far. It then computes a collection $Q_{r}$ of disjoint, small augmenting structures with respect to the current solution $B_{r}$, where the augmenting structures are composed of sets that have not been queried so far. It issues queries to these augmenting structures, and uses those that are found to exist to augment the current solution. The augmented solution is fed into the next round.

Similarly to our matching results, for any element $v \in U$, the number of sets that it belongs to and are also queried is at most $R$. Indeed, in each of the $R$ rounds, Algorithm 4 issues queries to disjoint augmenting structures, and each augmenting structure includes at most one set per element.

\subsection{Non-adaptive algorithm for $k$-set packing}

Once again, when going from the adaptive case to the non-adaptive case, the fraction of the omniscient optimum that we can obtain becomes significantly worse.

THEOREM 6.4. There exists a non-adaptive polynomial-time algorithm that, given a $k$-set instance $(U, A)$ and $\epsilon>0$, uses $O(n)$ queries overall and returns a $k$-set packing with expected cardinality $(1-\epsilon) \frac{(2 / k)^{2}}{2 / k+1} \bar{K}(A)$. 


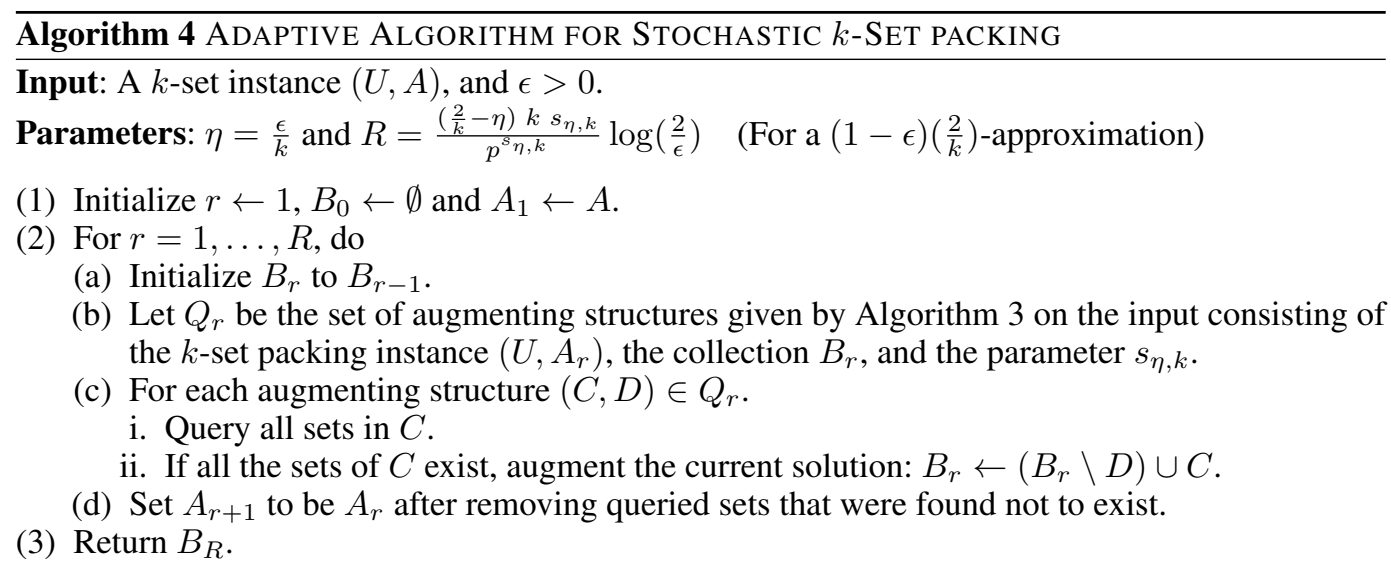

We present such a polynomial-time non-adaptive algorithm, Algorithm 5, that proceeds as follows. For $R$ rounds, at every round, using the local improvement algorithm of Hurkens and Schrijver [1989], we find a $\left(\frac{2}{k}-\eta\right)$-approximate solution to the $k$-set instance and remove it. Then, we query every set that is included in these $R$ solutions. We show that the expected cardinality of the maximum packing on the chosen sets is a $(1-\epsilon) \frac{(2 / k)^{2}}{2 / k+1}$ approximation of the expected optimal packing. As usual, it is easy to see that $O(n)$ queries are issued overall.

Importantly, the statements of Theorems 4.1 and 5.1 are special cases of the statements of Theorems 6.3 and 6.4, respectively, for $k=2$, although on a technical level the $k=2$ case must be handled separately (as we did, with less cumbersome terminology and technical constructions than in the general $k$-set packing setting).

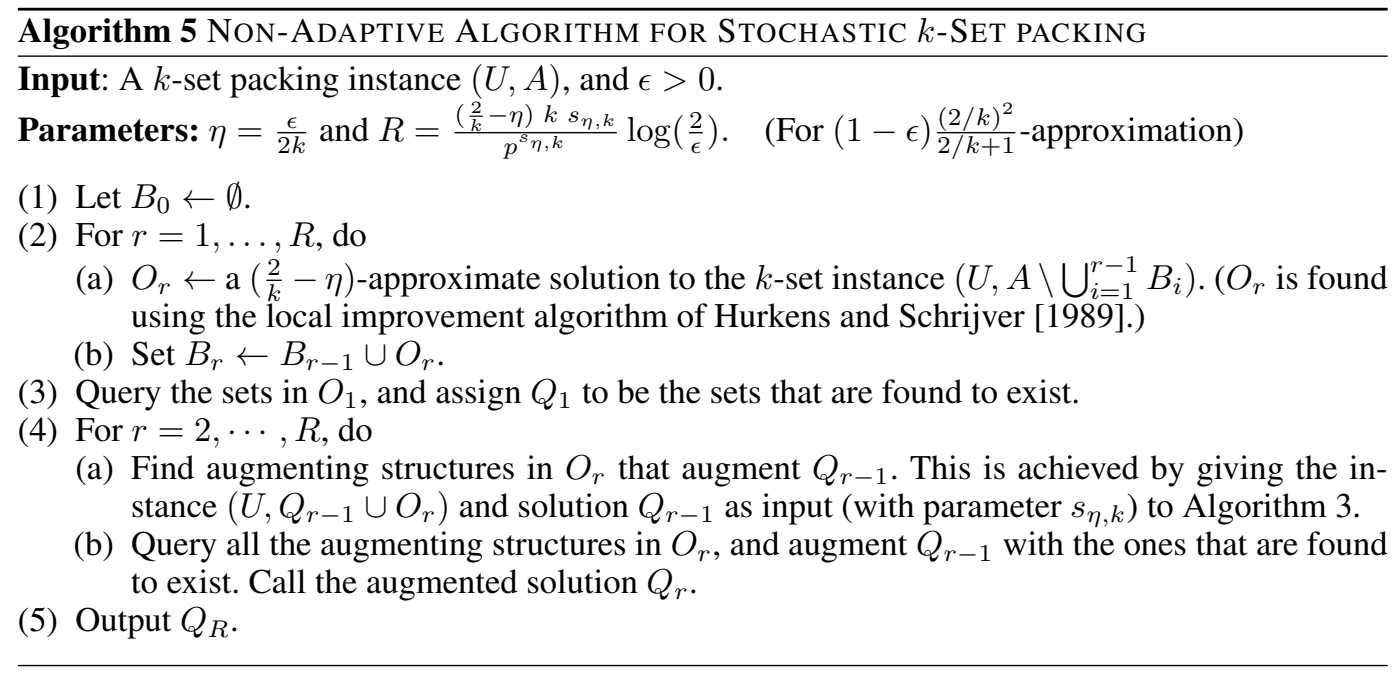

\section{EXPERIMENTAL RESULTS ON KIDNEY EXCHANGE COMPATIBILITY GRAPHS}

In this section, we support our theoretical results with empirical simulations from two kidney exchange compatibility graph distributions. The first distribution, due to Saidman et al. [2006], was designed to mimic the characteristics of a nationwide exchange in the United States in steady state. Fielded kidney exchanges have not yet reached that point, though; with this in mind, we also include results on real kidney exchange compatibility graphs drawn from the first 169 match runs 
of the UNOS nationwide kidney exchange. While these two families of graphs differ substantially, we find that even a small number $R$ of non-adaptive rounds, followed by a single period during which only those edges selected during the $R$ rounds are queried, results in large gains relative to the omniscient matching.

As is common in the kidney exchange literature, in the rest of this section we will loosely use the term "matching" to refer to both 2-set packing (equivalent to the traditional definition of matching, where two vertices connected by directed edges are translated to two vertices connected by a single undirected edge) and $k$-set packing, possibly with the inclusion of altruist-initiated chains.

This section does not directly test the algorithms presented in this paper. For the 2-cycles-only case, we do directly implement Algorithm 2. However, for the cases involving longer cycles and/or chains, we do not restrict ourselves to polynomial time algorithms (unlike in the theory part of this paper), instead choosing to optimally solve matching problems using integer programming during each round, as well as for the final matching and for the omniscient benchmark matching. This decision is informed by the current practice in kidney exchange, where computational resources are much less of a problem than human or monetary resources (of which the latter two are necessary for querying edges).

In our experiments, the planning of which edges to query proceeds in rounds as follows. Each round of matching calls as a subsolver the matching algorithm presented by Dickerson et al. [2013], which includes edge failure probabilities in the optimization objective to provide a maximum discounted utility matching. The set of cycles and chains present in a round's discounted matching are added to a set of edges to query, and then those cycles and chains are constrained from appearing in future rounds. After all rounds are completed, this set of edges is queried, and a final maximum discounted utility matching is compared against an omniscient matching that knows the set of non-failing edges up front.

\subsection{Experiments on dense generated graphs due to Saidman et al. [2006]}

We begin by looking at graphs drawn from a distribution due to Saidman et al. [2006], hereafter referred to as "the Saidman generator." This generator takes into account the blood types of patients and donors (such that the distribution is drawn from the general United States population), as well as three levels of PRA and various other medical characteristics of patients and donors that may affect the existence of an edge. Fielded kidney exchanges currently do not uniformly sample their pairs from the set of all needy patients and able donors in the US, as assumed by the Saidman generator; rather, exchanges tend to get hard-to-match patients who have not received an organ through other means. Because of this, the Saidman generator tends to produce compatibility graphs that are significantly denser than those seen in fielded kidney exchanges today (see, e.g., [Ashlagi et al. 2011, 2013]).

Figure 1 presents the fraction of the omniscient objective achieved by $R \in\{0,1, \ldots, 5\}$ nonadaptive rounds of edge testing for generated graphs with 250 patient-donor pairs and no altruistic donors, constrained to 2-cycles only (left) and both 2- and 3-cycles (right). Note that the case $R=0$ corresponds to no edge testing, where a maximum discounted utility matching is determined by the optimizer and then compared directly to the omniscient matching. The x-axis varies the uniform edge failure rate $f$ from 0.0 , where edges do not fail, to 0.9 , where edges only succeed with a $10 \%$ probability. Given an edge failure rate of $f$ in the figures below, we can translate to the $p$ used in the theoretical section of the paper as follows: 2 -cycles exists with probability $p_{2 \text {-cycle }}=(1-f)^{2}$, while a 3 -cycle exists with $p_{3 \text {-cycle }}=(1-f)^{3}$. For example, in the case of $f=0.9$, a 3 -cycle exists with very low probability $p=0.001$.

The utility of even a small number of edge queries is evident in Figure 1. Just a single round of testing $(R=1)$ results in $50.6 \%$ of omniscient - compared to just $29.8 \%$ with no edge testing - for edge failure probability $f=0.5$ in the 2 -cycle case, and there are similar gains in the 2 - and 3 -cycle case. For the same failure rate, setting $R=5$ captures $84.0 \%$ of the omniscient 2 -cycle matching and 

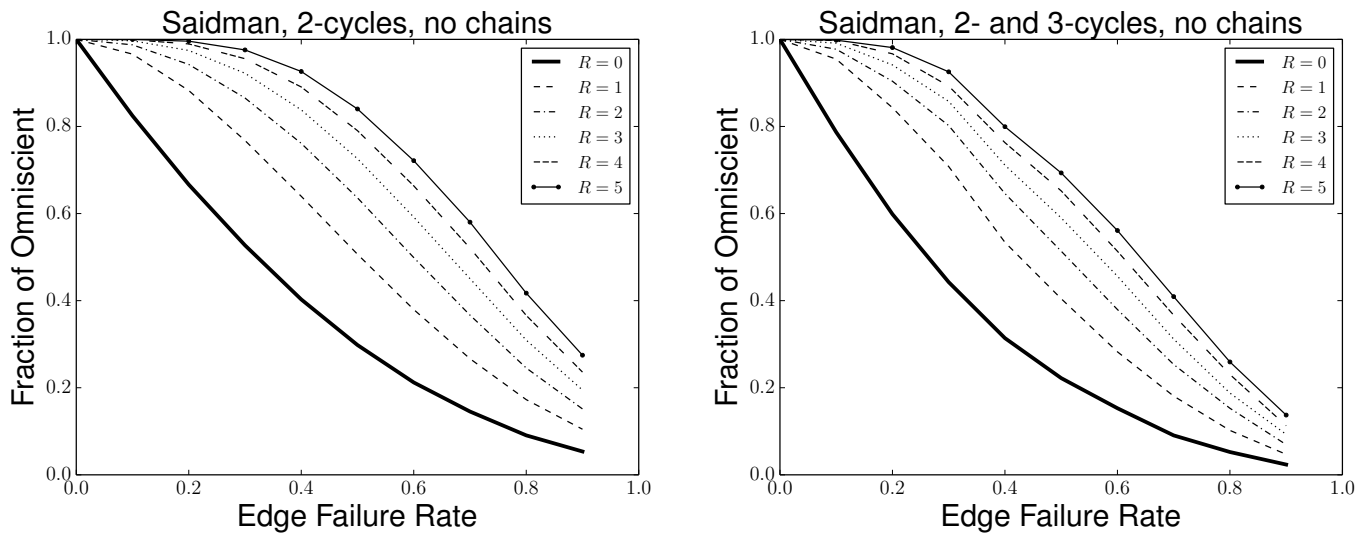

Fig. 1. Saidman generator graphs constrained to 2-cycles only (left) and both 2- and 3-cycles (right).

$69.3 \%$ in the 2 - and 3-cycle case-compared to just $22.2 \%$ when no edges are queried. Interestingly, we found no statistical difference between non-adaptive and adaptive matching on these graphs.

\subsection{Experiments on real match runs from the UNOS nationwide kidney exchange}

We now analyze the effect of querying a small number of edges per vertex on graphs drawn from the real world. Specifically, we use the first 169 match runs of the UNOS nationwide kidney exchange, which began matching in October 2010 on a monthly basis and now includes 143 transplant centers - that is, $60 \%$ of the centers in the U.S.- and performs match runs twice per week. These graphs, as with other fielded kidney exchanges [Ashlagi et al. 2013], are substantially less dense than those produced by the Saidman generator. This disparity between generated and real graphs has led to different theoretical results (e.g., efficient matching does not require long chains in a deterministic dense model [Ashlagi and Roth 2014; Dickerson et al. 2012b] but does in a sparse model [Ashlagi et al. 2011]) and empirical results (both in terms of match composition and experimental tractability [Constantino et al. 2013; Glorie et al. 2014; Anderson et al. 2015b]) in the past - a trend that continues here.

Figure 2 shows the fraction of the omniscient 2-cycle and 2-cycle with chains match size achieved by using only 2-cycles or both 2-cycles and chains and some small number of non-adaptive edge query rounds $R \in\{0,1, \ldots, 5\}$. For each of the 169 pre-test compatibility graphs and each of edge failure rates, 50 different ground truth compatibility graphs were generated. Chains can partially execute; that is, if the third edge in a chain of length 3 fails, then we include all successful edges (in this case, 2 edges) until that point in the final matching. More of the omniscient matching is achieved (even for the $R=0$ case) on these real-world graphs than on those from the Saidman generator presented in Section 7.1. Still, the gain realized even by a small number of edge query rounds is stark, with $R=5$ achieving over $90 \%$ of the omniscient objective for every failure rate in the 2 -cycles-only case, and over $75 \%$ of the omniscient objective when chains are included (and typically much more).

Figure 3 expands these results to the case with 2- and 3-cycles, both without and with chains. Slightly less of the omniscient matching objective is achieved across the board, but the overall increases due to $R \in\{1, \ldots, 5\}$ non-adaptive rounds of testing is once again prominent. Interestingly, we did not see a significant difference in results for adaptive and non-adaptive edge testing on the UNOS family of graphs, either.

We provide additional experimental results in the full version of this paper. Code to replicate all experiments is available at https://github.com/JohnDickerson/KidneyExchange; this codebase includes graph generators but, due to privacy concerns, does not include the real match runs from the UNOS exchange. 

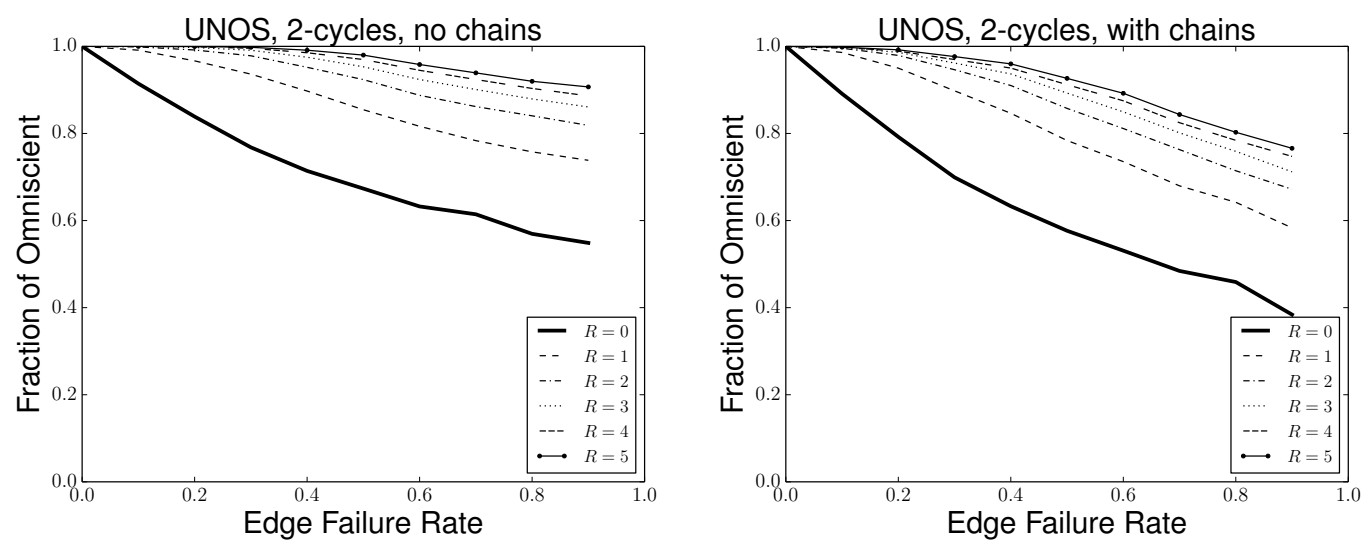

Fig. 2. Real UNOS match runs constrained to 2-cycles (left) and both 2-cycles and chains (right).
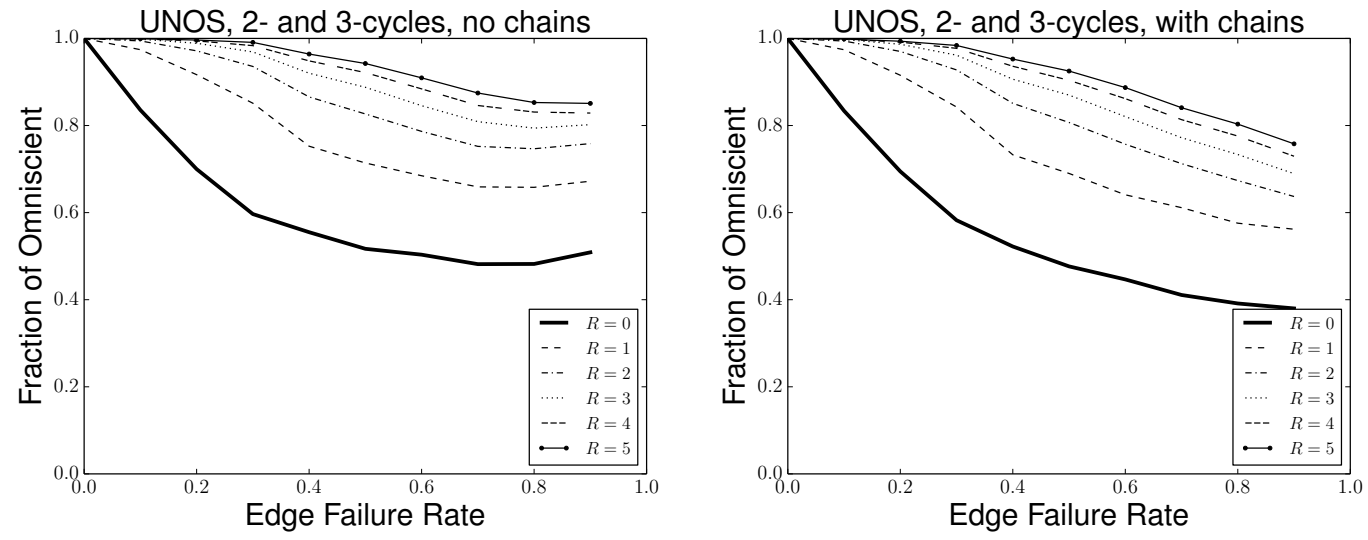

Fig. 3. Real UNOS match runs with 2- and 3-cycles and no chains (left) and with chains (right).

\section{CONCLUSIONS \& FUTURE RESEARCH}

In this paper, we addressed stochastic matching and its generalization to $k$-set packing from both a theoretical and experimental point of view. For the stochastic matching problem, we designed an adaptive algorithm that queries only a constant number of edges per vertex and achieves a $(1-\epsilon)$ fraction of the omniscient solution, for an arbitrarily small $\epsilon>0$-and performs the queries in only a constant number of rounds. We complemented this result with a non-adaptive algorithm that achieves a $(0.5-\epsilon)$ fraction of the omniscient optimum.

We then extended our results to the more general problem of stochastic $k$-set packing by designing an adaptive algorithm that achieves a $\left(\frac{2}{k}-\epsilon\right)$ fraction of the omniscient optimal solution, again with only $O(1)$ queries per element. This guarantee is quite close to the best known polynomial-time approximation ratio of $\frac{3}{k+1}-\epsilon$ for the standard non-stochastic setting [Fürer and Yu 2013].

We adapted these algorithms to the kidney exchange problem and, on both generated and real data from the first 169 runs of the UNOS US nationwide kidney exchange, explored the effect of a small number of edge query rounds on matching performance. In both cases-but especially on the real data - a very small number of non-adaptive edge queries per donor-patient pair results in large gains in expected successful matches across a wide range of edge failure probabilities. 


\subsection{Open theoretical problems}

Three main open theoretical problems remain open. First, our adaptive algorithm for the matching setting achieves a $(1-\epsilon)$-approximation in $O(1)$ rounds and using $O(1)$ queries per vertex. Is there a non-adaptive algorithm that achieves the same guarantee? Such an algorithm would make the practical message of the theoretical results even more appealing: instead of changing the status quo in two ways - more rounds of crossmatch tests, more tests per patient-we would only need to change it in the latter way.

Second, for both our adaptive and non-adaptive algorithms, the number of rounds $(R=$ $\left.\tilde{O}\left(p^{-1 / \epsilon}\right)\right)$ - even though independent of the number of donor-patient pairs - is exponential in $\frac{1}{\epsilon}$. On the other hand, our experiments show gains as high as $85 \%$ for even small values of $R \leq 5$. This leaves open an interesting question regarding the dependence of $R$ on the values of $p$ and $\epsilon$. Can a similar $1-\epsilon$ guarantee for general graphs be obtained using a number of rounds with better dependence on $p$ and $\epsilon$ ? If not, are there structural properties of kidney exchange graphs that we can exploit to achieve theoretical results with better dependence of $R$ on $p$ and $\epsilon$ ?

Third, for the case of $k$-set packing, we achieve a $\left(\frac{2}{k}-\epsilon\right)$-approximation using $O(n)$ queries-in polynomial time. In kidney exchange, however, our scarcest resource is crossmatch tests; computational hardness is circumvented daily, through integer programming techniques [Abraham et al. 2007]. Is there an exponential-time adaptive algorithm for $k$-set packing that requires $O(1)$ rounds and $O(n)$ queries, and achieves a $(1-\epsilon)$-approximation to the omniscient optimum? A positive answer would require a new approach, because ours is inherently constrained to constant-size augmenting structures, which cannot yield an approximation ratio better than $\frac{2}{k}-\epsilon$, even if we could compute optimal solutions to $k$-set packing [Hurkens and Schrijver 1989].

\subsection{Discussion of policy implications of experimental results}

Policy decisions in kidney exchange have been linked to economic and computational studies since before the first large-scale exchange was fielded in 2003-2004 [Roth et al. 2004, 2005]. A feedback loop exists between the reality of fielded exchanges-now not only in the United States but internationally as well-and the theoretical and empirical models that inform their operation, such that the latter has grown substantially closer to accurately representing the former in recent years. That said, many gaps still exist between the mathematical models used in kidney exchange studies and the systems that actually provide matches on a day-to-day basis.

More accurate models are often not adopted quickly, if at all, by exchanges. One reason for this is complexity - and not in the computational sense. Humans-doctors, lawyers, and other policymakers who are not necessarily versed in optimization or theoretical economics and computer science-and the organizations they represent rightfully wish to understand the workings of an exchange's matching policy. The techniques described in this paper are particularly exciting in that they are quite easy to explain in accessible language and they involve only mild changes to the status quo. At a high level, we are proposing to test some small number of promising potential matches for some subset of patient-donor pairs in a pool. As Section 7.2 shows, even a single extra edge test per pair will produce substantially better results.

Any new policy for kidney exchange has to address three practical restrictions in this space: (i) the monetary cost of crossmatches, (ii) the number of crossmatches that can be performed per person, as there is an inherent limit on the amount of blood that can be drawn from a person, and (iii) the time it takes to find the matches, as time plays a major role in the health of patients and crossmatches become less accurate as time passes and the results get old. For both our non-adaptive and adaptive algorithms, even a very small number of rounds $(R \leq 5)$ results in a very large gain in the objective. This is easily within the limits of considerations (i) and (ii) above. Our non-adaptive algorithm performs all chosen crossmatches in parallel, so the time taken by this method is similar to the current approach. Our adaptive algorithm, in practice, can be implemented by a one-time retrieval of $R$ rounds worth of blood from each donor-patient pair, then sending that blood to a central wet laboratory. Most crossmatches are performed via an "immediate spin", where the bloods are mixed 
together and either coagulate (which is bad) or do not (which is good). These tests are very fast, so a small number of rounds could be performed in a single day (assuming that tests in the same round are performed in parallel). Therefore, the timing constraint (iii) is not an issue for small $R$ (such as that used in our experiments) for the adaptive algorithm.

Clearly, more extensive studies would need to be undertaken before an exact policy recommendation could be made. These studies could take factors like the monetary cost of an extra crossmatch test or variability in testing prowess across different medical laboratories into account explicitly during the optimization process. Furthermore, various prioritization schemes could be implemented to help, for example, hard-to-match pairs find a feasible match by assigning them a higher edge query budget than easier-to-match pairs. The positive theoretical results presented in this paper, combined with the promising experimental results on real data, provide a firm basis and motivation for this type of policy analysis.

\section{REFERENCES}

Abraham, D. J., Blum, A., And SAndholm, T. 2007. Clearing algorithms for barter exchange markets: Enabling nationwide kidney exchanges. In Proceedings of the 8th ACM Conference on Electronic Commerce (EC). 295-304.

ADAMCZYK, M. 2011. Improved analysis of the greedy algorithm for stochastic matching. Information Processing Letters 111, 15, 731-737.

Akbarpour, M., Li, S., And Gharan, S. O. 2014. Dynamic matching market design. In Proceedings of the ACM Conference on Economics and Computation (EC). 355.

Anderson, R., Ashlagi, I., Gamarnik, D., And Kanoria, Y. 2015a. A dynamic model of barter exchange. In Proceedings of the 26th Annual ACM-SIAM Symposium on Discrete Algorithms (SODA). 1925-1933.

Anderson, R., Ashlagi, I., Gamarnik, D., And Roth, A. E. 2015b. Finding long chains in kidney exchange using the traveling salesman problem. Proceedings of the National Academy of Sciences 112, 3, 663-668.

As Adpour, A., NAZERZADEH, H., AND SABERI, A. 2008. Stochastic submodular maximization. In Proceedings of the 4th International Workshop on Internet and Network Economics (WINE). 477-489.

Ashlagi, I., Gamarnik, D., Rees, M. A., And Roth, A. E. 2011. The need for (long) chains in kidney exchange. Manuscript.

Ashlagi, I., Jaillet, P., And Manshadi, V. H. 2013. Kidney exchange in dynamic sparse heterogenous pools. In Proceedings of the 14th ACM Conference on Electronic Commerce (EC). 25-26.

Ashlagi, I. AND Roth, A. 2014. Free riding and participation in large scale, multi-hospital kidney exchange. Theoretical Economics. Forthcoming; preliminary version in EC'11.

AwASTHI, P. AND SANDHOLM, T. 2009. Online stochastic optimization in the large: Application to kidney exchange. In Proceedings of the 21st International Joint Conference on Artificial Intelligence (IJCAI). 405-411.

Bansal, N., Gupta, A., Li, J., Mestre, J., Nagarajan, V., And Rudra, A. 2012. When LP is the cure for your matching woes: Improved bounds for stochastic matchings. Algorithmica 63, 4, 733-762.

Blum, A., Gupta, A., Procaccia, A. D., and Sharma, A. 2013. Harnessing the power of two crossmatches. In Proceedings of the 14th ACM Conference on Electronic Commerce (EC). 123-140.

Bollobás, B. 2001. Random Graphs 2nd Ed. Cambridge University Press.

Chen, N., Immorlica, N., Karlin, A. R., Mahdian, M., And Rudra, A. 2009. Approximating matches made in heaven. In Proceedings of the 36th International Colloquium on Automata, Languages and Programming (ICALP). 266-278.

Constantino, M., Klimentova, X., Viana, A., And Rais, A. 2013. New insights on integer- 
programming models for the kidney exchange problem. European Journal of Operational Research 231, 1, 57-68.

Costello, K. P., Tetali, P., And Tripathi, P. 2012. Matching with commitment. In Proceedings of the 39th International Colloquium on Automata, Languages and Programming (ICALP). 822-833.

Dean, B. C., Goemans, M. X., And VondraK, J. 2004. Approximating the stochastic knapsack problem: The benefit of adaptivity. In Proceedings of the 45th Symposium on Foundations of Computer Science (FOCS). 208-217.

Dickerson, J. P., Procaccia, A. D., And Sandholm, T. 2012a. Dynamic matching via weighted myopia with application to kidney exchange. In Proceedings of the 26th AAAI Conference on Artificial Intelligence (AAAI). 1340-1346.

Dickerson, J. P., Procaccia, A. D., And SAndholm, T. 2012b. Optimizing kidney exchange with transplant chains: Theory and reality. In Proceedings of the 11th International Conference on Autonomous Agents and Multi-Agent Systems (AAMAS). 711-718.

Dickerson, J. P., Procaccia, A. D., And SAndholm, T. 2013. Failure-aware kidney exchange. In Proceedings of the 14th ACM Conference on Electronic Commerce (EC). 323-340.

Dickerson, J. P. AND SANDholm, T. 2015. FutureMatch: Combining human value judgments and machine learning to match in dynamic environments. In Proceedings of the 29th AAAI Conference on Artificial Intelligence (AAAI).

FÜRER, M. AND YU, H. 2013. Approximate the $k$-set packing problem by local improvements. CoRR abs/1307.2262.

Glorie, K. M., VAn DE Klundert, J. J., And Wagelmans, A. P. M. 2014. Kidney exchange with long chains: An efficient pricing algorithm for clearing barter exchanges with branch-andprice. Manufacturing \& Service Operations Management 16, 4, 498-512.

Goel, G. AND TRipathi, P. 2012. Matching with our eyes closed. In Proceedings of the 53rd Symposium on Foundations of Computer Science (FOCS). 718-727.

Gupta, A., Krishnaswamy, R., Nagarajan, V., And RaVi, R. 2012. Approximation algorithms for stochastic orienteering. In Proceedings of the 23rd Annual ACM-SIAM Symposium on Discrete Algorithms (SODA). 1522-1538.

Gupta, A. AND NAgARAJAN, V. 2013. A stochastic probing problem with applications. In Proceedings of the 16th Conference on Integer Programming and Combinatorial Optimization (IPCO). 205-216.

Hurkens, C. A. J. And SchriJver, A. 1989. On the size of systems of sets every $t$ of which have an SDR, with an application to the worst-case ratio of heuristics for packing problems. SIAM Journal on Discrete Mathematics 2, 1, 68-72.

Leishman, R., Formica, R., Andreoni, K., Friedewald, J., Sleeman, E., Monstello, C., Stewart, D., and Sandholm, T. 2013. The Organ Procurement and Transplantation Network (OPTN) Kidney Paired Donation Pilot Program (KPDPP): Review of current results. In American Transplant Congress (ATC). Talk abstract.

Manlove, D. And O’Malley, G. 2015. Paired and altruistic kidney donation in the UK: Algorithms and experimentation. ACM Journal of Experimental Algorithmics. To appear.

Roth, A. E., SÖnmez, T., And ÜnVER, M. U. 2004. Kidney exchange. Quarterly Journal of Economics 119, 2, 457-488.

Roth, A. E., Sönmez, T., And Ünver, M. U. 2005. Pairwise kidney exchange. Journal of Economic Theory 125, 151-188.

Roth, A. E., SÖNmeZ, T., AND ÜnVER, M. U. 2007. Efficient kidney exchange: Coincidence of wants in markets with compatibility-based preferences. American Economic Review 97, 3, 828-851.

Saidman, S. L., Roth, A. E., Sönmez, T., Ünver, M. U., and Delmonico, F. L. 2006. Increasing the opportunity of live kidney donation by matching for two and three way exchanges. Transplantation 81, 773-782.

ÜNVER, M. U. 2010. Dynamic kidney exchange. Review of Economic Studies 77, 1, 372-414. 